\title{
SPATIAL CORRELATION OF IMPURITY CHARGES INDUCED BY COULOMB INTERACTIONS - APPLICATION TO DX CENTERS IN GaAs
}

\author{
J. Kossut, Z. Wilamowski, T. Dietl and K. Świątek \\ Institute of Physics, Polish Academy of Sciences, \\ Al. Lotników 32/46, 02-668 Warsaw, Poland
}

(Received August 8, 1990)

\begin{abstract}
The electron mobility enhancement observed in heavily doped GaAs under hydrostatic pressure is interpreted in terms of spatial correlation between the donor charges within partially occupied system of impurities induced by strong inter-donor Coulomb interaction. A simple analytic theory is given for both $\mathrm{DX}^{0}$ and $\mathrm{DX}^{-}$models of the impurity state. The mobility is shown to increase together with pressure in both models. Estimates of the energy of the DX level are strongly perturbed by the inter-donor Coulomb interactions.
\end{abstract}

PACS numbers: 72.20.Dp, 72.80.Ey, 71.55.Eq

\section{Introduction}

It has been recently observed that the electron mobility in $\mathrm{HgSe}: \mathrm{Fe}$ at low temperatures may acquire anomalously high values [1-3]. The enhancement of the mobility occurs in the mixed valence regime when neutral $\mathrm{Fe}^{2+}$ and ionized $\mathrm{Fe}^{3+}$ states of $\mathrm{Fe}$ donor (whose energy is resonant with the conduction band of $\mathrm{HgSe}$ ) coexist. This regime is reached either by doping with $\mathrm{Fe}$ to the levels exceeding $\approx$ $5 \times 10^{18} \mathrm{~cm}^{-3}$ or, in samples with smaller amount of Fe donors, by application of the hydrostatic pressure [4]. Following the hypothesis put forward by Jerzy Mycielski [5] we were able to describe quantitatively the mobility enhancement by calculating the momentum relaxation time resulting from the scattering of the conduction electrons from the donor charges whose positions were not random but spatially correlated $[6,7]$. The correlation has its source in the Coulomb repulsion between the charges in the partially occupied donor system. As a result of this repulsion there exists a region surrounding each ionized donor where no other ionized donors reside. The correlation may be viewed as an effect precursory to formation of 
Wigner-like glassy state of charges localized on impurities. The scattering centers whose spatial positions are correlated yield longer relaxation times than randomly distributed scatterers - part of the scattering events in the former case have a coherent character and neither relax the momentum nor shorten the electron lifetime.

It has been also very recently realized $[8,9]$ that the behavior exhibited by low temperature mobility as a function of the hydrostatic pressure (applied at elevated temperature) [10] in heavily doped GaAs containing DX centers resembles the behavior exhibited by $\mathrm{HgSe}: \mathrm{Fe}$ - by lowering the energy of resonant impurity state by means of the pressure $p$ one brings the Fermi level into coincidence with the DX level. Then, some of the electrons, which at $p=0$ occupy the conduction band states, are captured at the DX centers and the concentration of band carriers is seen to drop. At the same time the mobility shows an increasing trend with pressure $[9,10]$.

The analogy between GaAs with DX centers and $\mathrm{HgSe}: \mathrm{Fe}$ is complete if the model describing the DX state in GaAs is that of a singly occupied state, known also as $\mathrm{DX}^{0}$ or $U>0$ model (where $U$ is the on-site electron-electron interaction). Recently, a concept of doubly occupied DX state was put forward (DX- or $U<0$ model) where a strong lattice distortion effect makes a double occupancy energetically more favorable $[11,12]$. The correlation induced by the inter-donor Coulomb interaction that have to be considered in the case of $\mathrm{DX}^{-}$ model is of a different nature. Namely, a positively charged ionized donor $d^{+}$tends to have a negatively charged occupied donor $\left(\mathrm{DX}^{-}\right)$in its immediate vicinity, thus forming a dipole-like object that scatters conduction electrons less effectively than two independent charged centers, provided that the arm of such dipole is shorter than the de Broglie's wavelength of the conduction electrons [13]. Correlation of impurity charges analogous to that occurring in the $\mathrm{DX}^{-}$model, were made responsible [14] for anomalously high values of the mobility (as well as its unexpectedly strong temperature dependence) observed in zero-gap $\mathrm{HgMnTe}[15,16]$ and $\mathrm{HgCdTe}[17,18]$ with the Fermi level trapped at the resonant acceptor state.

It is still a matter of debate which of the two models, $\mathrm{DX}^{0}$ or $\mathrm{DX}^{-}$, describes better the observed properties of DX centers (it is not unlikely that both $\mathrm{DX}^{0}$ and $\mathrm{DX}^{-}$states may even coexist). Therefore, both will be considered on an equal footing in this presentation. In fact, an attempt was made to distinguish between the two models using arguments involving analysis of the mobility vs. pressure data [19]. The analysis [19], however, did not consider the correlations mentioned above. As we shall show here they are of primary importance and when a proper account of their existence is made the conclusions reached in [19] are no longer valid [8].

In this paper we take the inter-donor correlation into account explicitly (not, as suggested in [20], by means of an effective screening) by considering a simple, though quite realistic, pair correlation function $g(r)$ within a short range correlation model. The calculations will be carried out for both $\mathrm{DX}^{-}$and $\mathrm{DX}^{0}$ models. 


\section{Short range correlation model}

We shall assume that the temperature at which the correlation sets in is very low and it is fair to put $T=0$. The validity of this simplification is discussed further in the paper.

\section{$D X^{0}$ model}

According to this model the DX impurity exists in two charge states: neutral $\mathrm{DX}^{0}$ state when occupied by an electron and positively charged $d^{+}$state when ionized. We choose the pair correlation function $g(r)$ of $d^{+}$centers to have a simple step like form: $g(r)=0$ for $r$ smaller than a certain correlation radius $r_{c}$ (to be determined later) and $g(r)=1$ for $r$ greater than $r_{c}--$ see Fig.1a. The pair
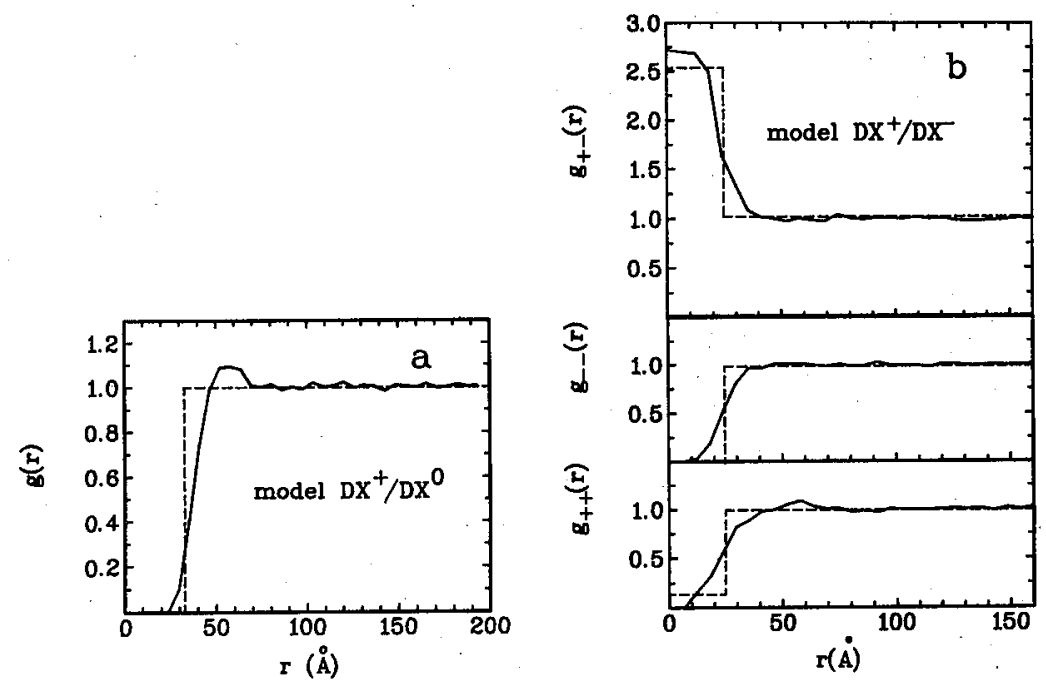

Fig.1. Pair correlation functions describing distribution of various impurity charges in GaAs with $N=1.1 \times 10^{19} \mathrm{~cm}^{-3}$ at $p=15 \mathrm{kbar}$ as given by the short range correlation model (dashed lines) and computer simulations ([21] - solid lines) for (a) DX model and (b) $\mathrm{DX}^{-}$model.

correlation function reflects probability of finding an ionized donor at a distance $r$ from a site where there already is a given ionized donor. With this in mind we see that the above form implies that there is a sphere around each ionized donor where there are no other ionized donors. At greater distances $g(r)=1$ means that we neglect existence of any correlations for $r>r_{c}$. It is not difficult to find an equation relating the correlation radius to the concentrations of DX centers $N$ and conduction electrons $n[7,21]$

$$
n V_{c}=1-\exp \left(-N V_{c}\right)
$$


where $V_{c}=4 \pi r_{c}^{3} / 3$. For $V_{c} N<<1$ one can approximate the solution of Eq.(1) by

$$
r_{c}=\left[\frac{3}{2 \pi N}\left(1-\frac{n}{N}\right)\right]^{\frac{1}{3}} .
$$

The pair correlation function is related to the structure factor $S(q)$ that, in turn, is needed in calculation of the momentum relaxation time due to the scattering from the charged centers

$$
\frac{1}{\tau}=\frac{N_{s c} m^{*}\left(E_{\mathrm{F}}\right)}{3 \pi^{2} \hbar^{3} n} \int_{0}^{2 k_{F}} q^{3} S(q)|V(q)|^{2} \mathrm{~d} q,
$$

where we assumed degenerate electron statistics, and where

$$
V(q)=4 \pi e^{2} /\left[\kappa\left(q^{2}+\lambda^{-2}\right)\right] .
$$

The screening of the Coulomb potentials of the ionized donors by the conduction electron gas is described by the screening radius $\lambda$ which in the Thomas-Fermi approximation is taken as $\left[\left(\pi \kappa \hbar^{2}\right) /\left(4 e^{2} k_{F} m^{*}\left(E_{\mathrm{F}}\right)\right]^{1 / 2}(\kappa\right.$ is the dielectric constant of GaAs, $m^{*}\left(E_{F}\right)$ - the effective mass at the Fermi level, and $k_{F}$ denotes the Fermi wave vector). For $\mathrm{DX}^{0}$ model, the concentration of scatterers, $N_{s c}$ in Eq.(3), is equal to the concentration of conduction electrons $n$. The mentioned relation between $g(r)$ and $S(q)$ is given by the Fourier transform

$$
S(q)=1+\frac{4 \pi n}{q} \int_{0} r \sin (q r)[g(r)-1] \mathrm{d} r .
$$

Using the simple form of $g(r)$ defined above one obtains

$$
S(q)=1-\frac{4 \pi n}{q^{3}}\left[\sin \left(q r_{c}\right)-q r_{c} \cos \left(q r_{c}\right)\right] .
$$

Inserting this result in Eq.(3) and performing the integration numerically we obtain $1 / \tau$ which when used to calculate the mobility results in the curve labeled (a) in Fig.2. For the sake of comparison we also show there (curve (b)) the values of the mobility obtained when no correlation of the scattering charges is present, i.e., for $S(q)=1$. The correlation does indeed result in an increase of the mobility as the number of electrons transferred from the conduction band to DX centers increases. The increase is much stronger than that in the case without correlations when it is solely due to changes of the screening parameter and the nonparabolicity of the conduction band [22].

Let us consider now the total energy (per unit volume) of the system consisting of the electrons in the conduction band and on DX centers. We have

$$
E_{\mathrm{Tot}}(n)=E_{\mathrm{Kin}}+E_{\mathrm{X}}+E_{\mathrm{DX}}(N-n)+E_{\mathrm{C}}
$$

where $E_{\text {Kin }}$ represents kinetic energy of the conduction electrons. The second term in Eq.(6) is the exchange correlation energy of the conduction electron gas which we take in the textbook form [23]

$$
E_{\mathrm{X}}=n\left(-0.916 / r_{s}+0.062 \ln r_{s}-0.096\right) R y^{*},
$$




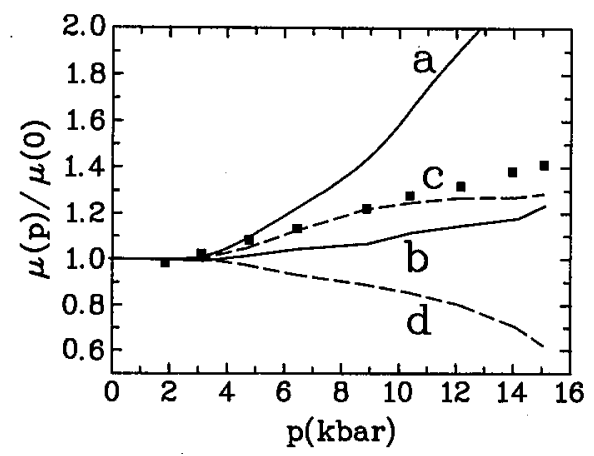

Fig.2. Relative mobility as a function of hydrostatic pressure in GaAs:Si with $N=$ $1.1 \times 10^{19} \mathrm{~cm}^{-3}$ : experimental points from [10], theoretical lines calculated within the short range correlation model - (a) for $\mathrm{DX}^{0}$ and (c) $\mathrm{DX}^{-}$, lines calculated without accounting for the correlations - (b) $\mathrm{DX}^{0}$ and (d) $\mathrm{DX}^{-}$.

with $r_{s}^{3}=3 /\left(4 \pi n a_{B}^{* 3}\right), a_{B}^{*}$ and $R y^{*}$ being the effective Bohr radius and effective rydberg, respectively. $E_{\mathrm{Dx}}$ in Eq.(6) stands for the energy of electrons occupying the DX state. The inter-donor Coulomb repulsion $E_{\mathrm{C}}$ can be calculated in terms of the pair correlation function

$$
E_{\mathrm{C}}=\frac{1}{2} n^{2} \int \exp (-r / \lambda) \frac{e^{2}}{\kappa r}[g(r)-1] \mathrm{d}^{3} r
$$

Note that the Coulomb interactions between completely delocalized conduction electrons cancel exactly the interactions between those electrons and charged donors. Using the piece-wise constant form of $g(r)$ defined above we obtain

$$
E_{\mathrm{C}}=-\frac{2 \pi e^{2}}{\kappa} n^{2} \lambda^{2}\left[1-\exp \left(-r_{\mathrm{c}} / \lambda\right)\left(1+r_{c} / \lambda\right)\right]
$$

By demanding that $\partial E_{\text {Tot }} / \partial n=0$ we derive the relation between $n$ and $N$ which can be used to extract the value of $E_{\mathrm{Dx}}$ (and its pressure coefficient) by fitting the solutions to the experimental $n$ vs. $p$ dependencies. A result of such fittings is shown in Fig. 3 by solid lines. An important property of $E_{\mathrm{DX}}$ values determined in this way is that they are common for all samples doped with a given species of a dopant. This is in contrast to the values obtained without considering the inter-donor Coulomb interactions when the $E_{\mathrm{DX}}$ were found to depend on the concentration of DX centers [10].

\section{$D X^{-}$model}

In the $\mathrm{DX}^{-}$model the DX impurity can exist in two states: empty $\left(d^{+}\right)$state and doubly occupied state $\left(\mathrm{DX}^{-}\right)$. Both are charged (respectively, positively and negatively) and, therefore, both scatter conduction electrons. Thus, to account for 


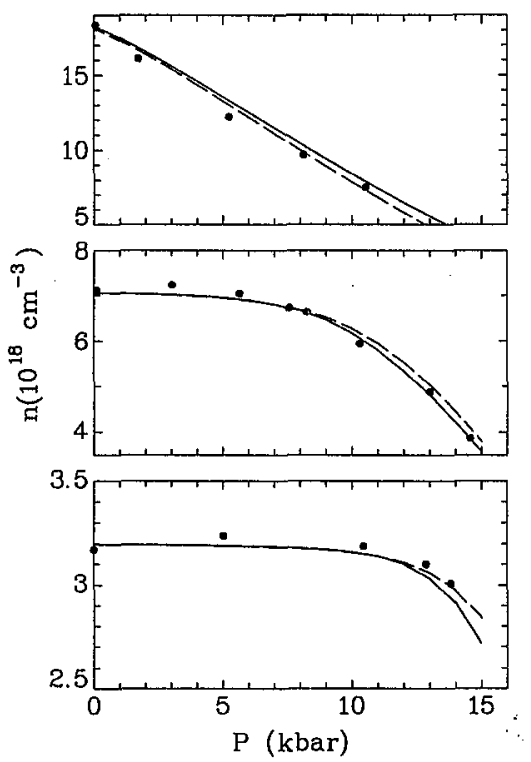

Fig.3. Conduction electron concentration as a function of hydrostatic pressure in GaAs doped with Sn: experimental points from [10], curves are fitted within the short range correlation model with $E_{\mathrm{DX}}=300 \mathrm{meV}$ and $\mathrm{d} E_{\mathrm{Dx}} / \mathrm{d} p=-14 \mathrm{meV} / \mathrm{kbar}$ assuming $\mathrm{DX}^{0}$ state (solid curves) and $E_{\mathrm{DX}}=320 \mathrm{meV}$ and $\mathrm{d} E_{\mathrm{Dx}} / \mathrm{d} p=-14 \mathrm{meV} / \mathrm{kbar}$ assuming $\mathrm{DX}^{-}$state (broken line).

correlations between all types of donors we need three pair correlation functions: $g_{++}(r), g_{--}(r)$, and $g_{+-}(r)=g_{-+}(r)$, where the subscripts correspond to the sign of charge localized on a given center forming the pair. Formation of dipoles invoked in the Introduction is described by $g_{+-}(r)$. These correlation functions are not independent. Since the dopants are distributed randomly in the crystal lattice the properly weighted correlation functions must add up to unity,

$$
\left(\frac{N_{+}}{N}\right)^{2} g_{++}(r)+2 \frac{N_{+} N_{-}}{N^{2}} g_{+-}(r)+\left(\frac{N_{-}}{N}\right)^{2} g_{--}(r)=1,
$$

where $N_{+}$and $N_{-}$are concentrations of empty and occupied DX centers

$$
N_{+}=(N+n) / 2 \text { and } N_{-}=(N-n) / 2 \text {. }
$$

As in the previous section we shall take the pair correlation functions as piece-wise constant, as shown in Fig.1b. Again, by considering the probability of finding various types of donors (empty or occupied) within the volume $V_{d}=4 \pi r_{d}^{3} / 3$ defined as containing only one negatively charged $\mathrm{DX}^{-}$center one finds in analogy to Eq.(1)

$$
N_{-} V_{d}=1-\exp \left(-N V_{d}\right)\left(1+N V_{d}\right) \text {. }
$$


Retaining only terms linear in $N V_{d}$ one finds the solutions of Eq.(11), in analogy to Eq.(2)

$$
r_{d}=\left[\frac{3}{4 \pi N}\left(1-\frac{n}{N}\right)\right]^{1 / 3} .
$$

One can interpret $r_{d}$ as a maximum lenght of a dipole that forms due to Coulomb attraction between $\mathrm{DX}^{-}$and $d^{+}$centers. Values of various $g^{\prime}$ 's for $r<r_{d}$ can be determined by making simple considerations involving probabilities of finding a donor in a given charge state within the volume $V_{d}$ surrounding a central donor. One obtains in this fashion

$$
\begin{aligned}
& g_{++}=\frac{1}{\left(N_{+} V_{d}\right)^{2}}\left[\alpha^{2}+2\left(\alpha-1+e^{-\alpha}\right)\right], \\
& g_{+-}=g_{-+}=\frac{1}{\left(N_{+} N_{-} V_{d}^{2}\right)}\left(\alpha-1+e^{-\alpha}\right),
\end{aligned}
$$

where $a=N V_{d}$. Using the above two expressions and the sum rule, Eq.(10), one concludes that $g_{--}=0$ for $r<r_{d}$.

Having thus determined all relevant pair correlation functions we may now proceed to calculate the momentum relaxation time yielded by scattering from variously charged donors. Elementary considerations lead to conclusion that $S(q)$ to be inserted in Eq.(3) reads in the case of $\mathrm{DX}^{-}$model

$$
S(q)=1-\frac{4 \pi}{q^{3}}\left[\sin \left(q r_{d}\right)-q r_{d} \cos \left(q r_{d}\right)\right]\left\{\frac{n^{2}}{N}-N\left[1-\frac{4}{\alpha^{2}}\left(\alpha-1+e^{-\alpha}\right)\right]\right\} .
$$

The mobility resulting from numerical integration of Eq.(3) with the above form of $S(q)$ is presented in Fig.2 by the curve labeled (c). It shows (contrary to that obtained when the correlations are neglected in the $\mathrm{DX}^{-}$model - curve (d) in Fig.2) an increasing trend as the pressure is increased.

The donor-donor Coulomb interaction energy needed to calculate the total energy can again be expressed in terms of the pair correlation functions. Using Eq.(13) and (14) we obtain

$$
E_{\mathrm{C}}=-\frac{2 \pi e^{2}}{\kappa} \lambda^{2}\left[1-\exp \left(-r_{d} / \lambda\right)\left(1+\frac{r_{d}}{\lambda}\right)\right]\left(N^{2}+n^{2}\right) .
$$

Demanding that the total energy is at the minimum we obtain again the relation between $n$ and $N$. Fitting the values of $E_{\mathrm{DX}}$ and its pressure coefficient in this relationship we can reproduce the experimental $n$ vs. $p$ behavior. As in the case of $\mathrm{DX}^{0}$ model we obtained a fair description of this behavior with a single value of $E_{\mathrm{DX}}$ for all samples doped with a given element, see, Fig.3.

\section{Discussion}

As seen from Fig. 3 it is impossible to discern which of the two competing models of the DX state is correct basing oneself on the $n$ vs. $p$ data obtained in 
heavily doped GaAs samples. Neither it is possible to distinguish between them on the basis of the $\mu$ vs. $p$ relationships - with inter-donor Coulomb interactions both models predict an increase of the mobility with pressure in the region where $n$ is diminished by capture on DX centers.

The applicability of the extremely simple form of the pair correlation function used in the previous Sections is confirmed by our computer simulations of the system. The correlation functions obtained by such simulations are shown in Fig.1 by solid lines. The simulated curves show features which resemble those of liquid substances.

Let us comment now on the legitimacy of the zero-temperature approach used in our presentation. In reality, in GaAs the correlation is frozen at $T \approx 100 \mathrm{~K}$. This temperature corresponds to an energy $8.5 \mathrm{meV}$ and is still smaller than the gain in the Coulomb interaction energy (per one electron) due to correlation which, as calculated using Eq.(9) and Eq.(16), is $20.2 \mathrm{meV}$ in the case of $\mathrm{DX}^{-}$model and $10.3 \mathrm{meV}$ in the case of $\mathrm{DX}^{0}$ model for $N=1.1 \times 10^{19} \mathrm{~cm}^{-3}$ sample at $p=10 \mathrm{kbar}$. So, the temperature does not destroy the correlation completely at the freeze-in temperature, particularly in the $\mathrm{DX}^{-}$model. Our estimates of the degree of the correlation do, however, possess a character of an "upper limit" estimate and the real correlation is certainly less pronounced (therefore our caution in regard to drawing quantitative conclusions concerning which of the two models describes better the DX state in GaAs).

\section{Concluding remarks}

We have shown that under conditions of incomplete occupancy of the donor states a spatial correlation of donor charges, due to mutual Coulomb interaction, appears. It results in an enhancement of the mobility compared to that expected for random distribution of the scattering potentials. We showed here that this mechanism is compatible with observations in GaAs containing DX centers subject to hydrostatic pressures. As mentioned the correlation of donor charges and accompanying increase of the mobility is also observed in other systems of which $\mathrm{HgSe}: \mathrm{Fe}$ is the most notable. Without attempting to give an exhaustive list let us mention also $\mathrm{HgMnTe}$ and $\mathrm{HgCdTe}$ with resonant acceptors already quoted in this context, quasi two-dimensional conducting layers of delta-doped GaAs [25] and, probably, $\mathrm{PbTe}: \mathrm{Cr}[26]$. It is, therefore, not unlikely that use of resonant impurity states will turn to be a general method of obtaining semiconducting materials with mobility superior to that in samples with shallow hydrogen-like impurity states.

\section{Acknowledgements}

We are grateful to T. Suski, J.M. Langer and W. Walukiewicz for stimulating discussions. 


\section{References}

[1] F. Pool, J. Kossut, U. Debska, R. Reifenberger, Phys. Rev. B 35, 3900 (1987).

[2] W. Dobrowolski et al., in Proc. 18th Internat. Conf. Phys. Semicond. (Stockholm 1986), ed. O. Engstrom, World Scientific, Singapore 1986, p.1743.

[3] For reviews of experimental results obtained in $\mathrm{HgSe}: \mathrm{Fe}$, see, e.g., A. Mycielski, J. Appl. Phys. 63, 3279 (1988), R. Reifenberger, J. Kossut, J. Vac. Sci. Technol. A 5, 2995 (1987).

[4] C. Skierbiszewski et al, Semicond. Sci. Technol. 4, 293 (1989).

[5] J. Mycielski, Solid State Commun. 60, 165 (1986).

[6] J. Kossut, W. Dobrowolski, T. Dietl, Z. Wilamowski, K. Świątek, Semicond. Sci. Technol. 5, S260 (1990).

[7] Z. Wilamowski, K. Świątek, T. Dietl, J. Kossut, Solid State Commun. 73, 833 (1990).

[8] T. Dietl, L. Dmowski, J. Kossut, E. Litwin- Staszewska, T. Piotrzkowski, T. Suski, K. Światek, Z. Wilamowski, Acta Phys. Pol. A77, 29 (1990).

[9] T. Suski, P. Wisniewski, E. Litwin-Staszewska, J. Kossut, Z. Wilamowski, T. Dietl, K. Świątek, K. Ploog, J. Knecht, Semicond. Sci. Technol. 5, 261 (1990).

[10] D.K. Maude et al., Phys. Rev. Lett. 52, 821 (1987).

[11] D.J. Chadi, K.J. Chang, Phys. Rev. Lett. 61, 873 (1988); Phys. Rev. B 39, 10063 (1989).

[12] K. Khachaturyan, E.R. Weber, M. Kamińska, Material Science Forum, Vols. 38-48, Trans. Tech. Publications, (1989) p.1067.

[13] Similar ideas are also employed by E.P. O'Reilly, Appl. Phys. Lett. 55, 1409 (1989).

[14] N.B. Brandt et al., Zh. Eksp. Teor. Fiz. 84, 1059 (1983) [Sov. Phys. JETP $57,614(1983)]$.

[15] R. Mani, J.R. Anderson, Phys. Rev. B 38, 3354 (1986).

[16] M. Sawicki et al., in Proc. Internat. Conf. Application of High Magnetic Fields in Physics of Semiconductors, Grenoble 1982, ed. G. Landwehr, Springer Verlag, Berlin, 1983 p. 382.

[17] G. Weil, A. Kozacki, C. Verie, in Physics of Narrow Gap Semiconductors, ed. J. Raułuszkiewicz, M. Górska, E. Kaczmarek, Polish Scientific Publishers, Warszawa 1978, p.173.

[18] N.B. Brandt et al., Fiz. Tekh. Poluprov. 17, 18 (1983) [Sov. Phys. Semicond. 17, 11 (1983)].

[19] D.K. Maude, L. Eaves, T.J. Foster, J.C. Portal, Phys. Rev. Lett. 62, 1922 (1989).

[20] D.J. Chadi, K.J. Chang, W. Walukiewicz, Phys. Rev. Lett. 62, 1923 (1989). 
[21] J. Kossut, Z. Wilamowski, T. Dietl, K. Świątek, to be published in Proc. 20th Internat. Conf. Phys. Semicond., Thessaloniki 1990.

[22] Derivation of two-dimensional version of Eq.(1) can be found in E.V. Chenskii, Yu.Ya. Tkach, Zh. Eksp. Teor. Fiz. 79, 1809 (1980).

[23] A. Raymond, J.L. Robert, C. Bernard, J. Phys. C (Solid State) 12, 2289 (1979).

[24] W. Jones, N.H. March, Theoretical Solid State Physics, Wiley-Interscience, London 1973.

[25] A. Zrenner, F. Koch, R.L. Williams, R.A. Stradling, K. Ploog, G. Weimann, Semicond. Sci. Technol. 3, 1203 (1988).

[26] B.A. Akimov et al., Fiz. Tekh. Poluprov. 23, 244 (1989). 